\title{
Counterexamples to Segal's Measure Representation Theorem
}

\author{
PETER WAKKER* \\ University of Nïmegen (NICI), P.O. Box 9104, 6500 HE Nijmegen, The Netherlands
}

\begin{abstract}
This article discusses relations between several notions of continuity in rank-dependent utility, and in the generalized version of rank-dependent utility as initiated by Segal. Primarily, examples are given to show logical independencies between these notions of continuity. This also leads to counterexamples to Segal's (1989) characterizing theorem 1.
\end{abstract}

Key words: rank-dependent utility, qualitative probability

The rank-dependent stream in the modern nonexpected utility models was initiated by Quiggin (1982) and Schmeidler (1989; first version, 1982). An often-cited paper is Segal (1989; first version, 1984). It was the first to introduce a generalization, called the measure representation approach, of Quiggin's rank-dependent model. In Quiggin's model, a lottery $\left(p_{1}, x_{1} ; \ldots ; p_{n}, x_{n}\right)$, yielding $\$ x_{j}$ with probability $p_{j}$ for each $j$, and with $x_{1} \geq \ldots \geq x_{n}$, is evaluated by a functional

$$
\sum_{i=1}^{n}\left[f\left(p_{1}+\ldots+p_{i}\right) u\left(x_{i}\right)-f\left(p_{1}+\ldots+p_{i-1}\right) u\left(x_{i}\right)\right] .
$$

Expected utility is the special case where the probability transformation function $f$ is the identity. The rank-dependent form allows a separation between attitudes to outcomes, modeled through $u$, and attitudes to probabilities, modeled through $f$. Segal's measure approach replaces $f\left(p_{1}+\ldots+p_{i}\right) u\left(x_{i}\right)$ above by a general function $V\left(p_{1}+\ldots+p_{i}, x_{i}\right)$; i.e., it deletes the separability into the factors $u$ and $f$. Later this model was also studied in Green and Jullien (1988), Chew and Epstein (1989), Chateauneuf (1990), Puppe (1990), and Chew and Wakker (1991).

Axiomatizations provide characterizations of quantitative models; i.e., they provide necessary and sufficient conditions for the applicability of quantitative models in terms of the observable primitive, the preference relation of a decision maker. Thus axiomatizations describe the empirical content of models and show their intrinsic consistency. Segal

*This article is a rewritten version of Wakker (1990a). Puppe (1990) independently discovered that Segal's (1989) theorem 1 is not correct. This research has been made possible by a fellowship of the Royal Netherlands Academy of Arts and Sciences. 
used geometric properties of epigraphs of distribution functions to describe and characterize models. This is mathematically useful, and makes possible the application of ideas from qualitative probability theory. As pointed out in Karni and Schmeidler (1990), a disadvantage is that it lacks a clear behavioral interpretation. A characterization of the general measure approach by geometric conditions is provided in Segal's (1989) theorem 1. The other results in his paper are based on that theorem.

This article shows, however, that the theorem is not correct. Example 4 shows that Segal's conditions do not imply the existence of a representing measure. Further, the continuity claims in Segal's theorem are not correct. The intricacies of absolute continuity and of the popular weak continuity, as demonstrated by the examples, also complicate the analyses in Green and Jullien (1989), Chew and Epstein (1989), and Puppe (1990). Segal (1990, lemma 8) presents a new alternative characterization of his generalized model. While this is based on Segal (1989, theorem 1) (some other references are also given), it actually contradicts the latter because the absolute continuity conditions have been deleted. Also, this new alternative characterization is incorrect, as will be shown by the examples below. A correct theorem that avoids the problems indicated in this article will appear in Segal (1992). Correct results have also appeared in Chateauneuf (1990) and Chew and Wakker (1991, section 5). Further, Chew, Epstein, and Wakker (1991) provided corrections for Chew and Epstein (1989), and Quiggin and Wakker (1992) provided corrections for Quiggin (1982).

\section{Implications of the examples}

Notations, domains, and terminology in this article are as in Segal (1989); i.e., $\geqslant$ is a preference relation over $L$, the set of probability distributions over $[0, M]$, for some fixed $M>0$. As usual, we assume that all elements of $L$ are countably additive. For $X \in L, F_{X}$ denotes the distribution function, assigning to each $\alpha \in$ IR the probability under $X$ that $\alpha$ or less will result. Any probability distribution $X$ is uniquely related to the associated epigraph $X^{0}$ of the distribution function, i.e., the closure of the area in the square $[0, M] \times$ $[0,1]$ above the graph of $F_{X}$. Note that countable additivity of the elements of $L$ is essential for this unique relatedness. $L^{0}$ is the set of all epigraphs, and is identified with $L$. So $\geqslant$ can also be considered a preference relation on $L^{0}$. We assume throughout that Segal's measure-theoretic approach holds, i.e., $\geqslant$ is represented by a measure $v$ on the Borel subsets of $[0, M] \times[0,1] .{ }^{1}$ A modification appears in example 4 , where $v$ is allowed to take the value infinite. In the other examples, $v$ only takes finite values. Obviously, $\geqslant$ is transitive and complete in all examples, and is easily seen to satisfy Segal's irrelevance axiom -that is, $\left[X \geqslant Y\right.$ if and only if $\left.X^{\prime} \geqslant Y^{\prime}\right]$ whenever on a set $S$ that is a finite union of segments, $F_{X}=F_{Y}$ and $F_{X^{\prime}}=F_{Y^{\prime}}$, whereas $F_{X}=F_{X^{\prime}}$ and $F_{Y}=F_{Y^{\prime}}$ outside $S$. It can be seen that in the presence of the other conditions, the irrelevance axiom is equivalent to the stronger axiom that allows $S$ to be general, as well as to the weaker axiom that requires $X, Y, X^{\prime}, Y^{\prime}$ to be simple. Let us mention that the question of representability of preference relations on subsets ("events") by ("probability") measures is studied in qualitative probability theory; Fishburn (1986) gives a survey. The irrelevance axiom is 
the additivity axiom from qualitative probability theory (see Fishburn, 1986, p. 336; Segal, 1986), reformulated for the present context. Green and Jullien (1988) and Segal (1990, lemma 8) use, instead of irrelevance, an ordinal independence condition that by itself is weaker than irrelevance. In the presence of the usual conditions it can, however, be seen to be equivalent to irrelevance, mainly by Gorman's (1968) result; see Chew and Wakker (1991, section 5).

Table 1 summarizes the properties of the five examples of section 2 . We give the table immediately here, so that already at this stage the reader can infer the logical relations between several properties for preferences, and the implications of the examples for Segal's results. For elaborations the reader should consult section 2. Let us also mention that in all examples the representing measure $v$ is a ratio scale, i.e., is unique up to a positive scale factor. All properties considered below are invariant under variation of the scale factor; hence the problems below cannot be avoided by the choice of an alternative representing measure.

Finally, we give some preparatory definitions. As in Segal (1989), stochastic dominance is taken as strict. By $\lambda$ we denote the Lebesgue measure on $[0, M] \times[0,1]$. A measure $v$ is absolutely continuous with respect to a measure $w$ if $[w(A)=0 \Rightarrow v(A)=0]$ for all $A$. The distribution function of $v$ assigns the value $v((-\infty, \alpha] \times(-\infty, \beta])$ to each $(\alpha, \beta) \in \mathrm{IR}^{2}$.

Segal's (1989) theorem 1 claims that the conditions of stochastic dominance, irrelevance, and continuity with respect to weak convergence for $\geqslant$ are equivalent to the existence of a representing measure $v$ that is mutually absolutely continuous with respect to $\lambda$. Example 1, however, shows that absolute continuity of $v$ with respect to $\lambda$ is not implied by the other conditions. Example 2 shows that this is neither implied in the special case of RDU, where $v$ is a product measure. Further, this example shows that also the claimed absolute continuity of $\lambda$ with respect to $v$ is not implied by irrelevance, stochastic dominance, and weak convergence continuity. One might conjecture that these problems could be overcome by replacing the requirements of absolute continuity by the requirement that the distribution function of $v$ be continuous. Example 3 shows that this does not work, because then weak convergence continuity of $\geqslant$ need not be implied, even if stochastic dominance is.

Table 1. A summary of the properties of the five examples

\begin{tabular}{lllllllll}
\hline & $\begin{array}{l}\text { st.dom } \\
\text { for } \geqslant\end{array}$ & $\begin{array}{l}\text { irr. ax. } \\
\text { for } \geqslant\end{array}$ & $\begin{array}{l}\text { weak cont. } \\
\text { for } \geqslant\end{array}$ & $\begin{array}{l}v \text { ab.ct. } \\
\text { wrt. } \lambda\end{array}$ & $\begin{array}{l}\lambda \text { ab.ct. } \\
\text { wrt. } v\end{array}$ & $\begin{array}{l}v \\
\text { finite }\end{array}$ & $\begin{array}{l}\text { distr. fion } \\
\text { of } v \text { cont. }\end{array}$ & $\begin{array}{l}\text { RDU: } \\
\text { prod. meas. }\end{array}$ \\
\hline Ex.1 & + & + & + & - & + & + & + & - \\
Ex.2 & + & + & + & - & - & + & + & + \\
Ex.3 & + & + & - & - & + & + & + & - \\
Ex.4 & + & + & + & + & + & - & + ND $^{\mathrm{a}}$ & - \\
Ex.5 & - & + & - & - & - & + & - & + \\
\hline
\end{tabular}

a Not defined. 
Although Segal (1989) does not make explicit whether the measure $v$ is allowed to be extended (i.e., assign value $\infty$ ), the paper, in particular the proof of his theorem 2 , suggests that the value infinite is not intended to occur. Example 4 shows, however, that finiteness of $v$ is not implied by stochastic dominance, irrelevance, and weak convergence continuity, i.e., the conditions of Segal's theorem 1. Actually, all elements of $L^{0}$ (except the empty set) get assigned measure infinite in this example, and an alternative way to derive preferences from the measure must be invoked.

Segal's (1990) lemma 8 claims that the same conditions for a binary relation as considered above, i.e., stochastic dominance, irrelevance, ${ }^{2}$ and continuity with respect to weak convergence for $\geqslant$, are equivalent to the existence of a general representing measure $v$. The lemma does not impose absolute continuity, and makes explicit that the measure should only satisfy finite additivity (as opposed to countable additivity). Theorem 1 in Segal (1989) does not address finite/countable additivity of the measure; the proof only claims finite additivity, but the adopted extension process seems to require countable additivity. ${ }^{3}$ Example 3 gives a counterexample to Segal's (1990) lemma 8, by showing that the conditions for the measure $v$ do not imply weak convergence continuity for preferences. Atomic measures $v$ give alternative counterexamples, showing that also stochastic dominance can be violated (see example 5).

\section{Elaborations of the examples}

In examples 1 and 3 below, we shall set $v:=w / 2+\lambda /(2 M)$ for some measure $w$. Then $\lambda$ is absolutely continuous with respect to $v$. That in turn implies that $\geqslant$ satisfies (strict monotonicity with respect to first-order) stochastic dominance. Whenever we add "almost everywhere" to a statement, we mean that the statement holds true except for a set with Lebesgue measure 0 . By lup we denote the diagonal through the points $(0,0)$ and $(M, 1) ; v^{\text {up }}$ is a kind of uniform distribution concentrated on $1^{\text {up }}$, i.e., to every subset of $1^{\text {up }}$ the measure $v^{\text {up }}$ assigns the length of the subset divided by the length of lup, and $v$ up: $A \mapsto$ $v^{\text {up }}\left(A \cap \mathrm{l}^{\mathrm{up}}\right)$. Similarly, $\mathrm{l}^{\mathrm{dn}}$ denotes the diagonal through the points $(0,1)$ and $(M, 0)$; to every subset of $1^{\mathrm{dn}}$ the measure $v^{\mathrm{dn}}$ assigns the length of the subset divided by the length of $\mathrm{l}^{\mathrm{dn}}$, and $v^{\mathrm{dn}}: A \mapsto v^{\mathrm{dn}}\left(A \cap \mathrm{I}^{\mathrm{dn}}\right)$. The diagonal lup, a boundary of a probability distribution (the uniform distribution), is used in example 3 , where weak convergence continuity is violated; $l^{\mathrm{dn}}$ is used in example 1 , where weak convergence continuity is satisfied.

\subsection{Example 1}

Let $v:=v^{\mathrm{dn}} / 2+\lambda /(2 M)$. It is not absolutely continuous with respect to $\lambda$ because it assigns a positive measure to the Lebesgue null set $1^{\mathrm{dn}}$. The only property of table 1 that warrants further elaboration is weak convergence continuity of $\geqslant$. Suppose $Y_{i}$ converges weakly to $Y$. It suffices to show that $v\left(Y_{i}\right)$ converges to $v(Y)$. We show this for $\lambda$ and $v^{\text {dn }}$ instead of $v$. Convergence of $\lambda\left(Y_{i}\right)$ to $\lambda(Y)$ follows because $\lambda$ is countably additive and the 
$Y_{i}$ as sets converge to $Y$ as set almost everywhere: nonconvergence can only occur on the boundary of $Y$. (The proof of almost convergence is simplified if one also allows for nonconvergence on vertical lines related to discontinuity points of $F_{Y}$.) Convergence of $\lambda\left(Y_{i}\right)$ to $\lambda(Y)$ can also be shown by the Lebesgue convergence theorem applied to the indicator functions of the $Y_{i}$ 's, which converge pointwise to the indicator function of $Y$ almost everywhere. Also $v^{\mathrm{dn}}\left(Y_{i}\right)$ converges to $v^{\mathrm{dn}}(Y)$ : Suppose the boundary of $Y$ intersects $1^{\mathrm{dn}}$ in a point $p$; this point is uniquely determined. Then $v^{\mathrm{dn}}(Y)$ is the $v^{\mathrm{dn}}$ measure of $1^{\text {dn }}$ "to the left" of $p$. It is straightforwardly verified that the intersection points $p^{i}$ of $Y_{i}$ with $l^{\mathrm{dn}}$ converge to $p$ if $p$ is a continuity point of $F_{Y}$; also this can be shown if $p$ is not a continuity point of $F_{Y}$, be it in a somewhat more complicated manner (look at first coordinates of the $p^{i}$ ). Thus also the $v^{\mathrm{dn}}$ measure of the $Y_{i}$ 's, which is the measure of $1^{\mathrm{dn}}$ to the left of $p^{i}$, converges to the $v^{\mathrm{dn}}$ measure of $Y$.

\subsection{Example 2}

Let $v$ be the product measure of a probability measure $P$ on $[0, M]$, still to be described, and the (one-dimensional) Lebesgue measure $\lambda^{1}$ on $[0,1]$. Suppose that $P$ assigns measure 1 to a set with Lebesgue measure 0 , and that the distribution function of $P$ is continuous (so that $P$ has no atoms) and strictly increasing. For example, for the distribution function of $P$ the Cantor ternary function on $[0, M]$ (see Royden, 1963, problems 5.9 and 2.42) can be chosen. $P$ is not absolutely continuous with respect to $\lambda^{1}$, and $\lambda^{2}$ is not absolutely continuous with respect to $P$. Thus $v$ is not absolutely continuous with respect to $\lambda$, and neither is $\lambda$ with respect to $v$. Finally, we derive weak convergence continuity for $\geqslant$. Suppose $Y_{j}$ converges weakly to $Y$. It suffices to show that $v\left(Y_{j}\right)$ converges to $v(Y)$. By countable additivity of $v$, it suffices to show that the sets $Y_{i}$ converge to the set $Y$, except on a set with $v$-measure 0 . The set of nonconvergence, to be shown to have $v$-measure 0 , is a subset of the union of two sets, each with $v$-measure 0 . The first set is the set of points with as first coordinate a discontinuity of $F_{y}$. There are at most countably many such discontinuities, their $P$-measure is 0 (because $P$ has no atoms), and thus also the first set must indeed have $v$-measure 0 . The second set is the set of boundary points of $F_{Y}$ that do not have a first coordinate that is a discontinuity of $F_{Y}$. This set has $v$-measure 0 because it has everywhere "height" 0 , in the same way that a set with height $\varepsilon$ everywhere has $v$-measure $\varepsilon$ (e.g., by repeated integration of the indicator-function, first with respect to the second coordinate).

\subsection{Example 3}

Let $v:=v^{\text {up }} / 2+\lambda /(2 M)$. It is not absolutely continuous with respect to $\lambda$ because it assigns positive measure to the Lebesgue-null-set $l^{\text {up }}$. The distribution function of $v$ is continuous. In this example, $\geqslant$ is not continuous with respect to weak convergence: Let $Y \in L^{0}$ be the area above $1^{\text {up }}$, associated with the uniform distribution. Suppose $Y_{i}$ is such that, at each point of the domain, $F_{Y_{i}}$ decreases to $F_{Y}$, and such that at each point of 
the domain except 0 and $M, F_{Y_{\mathrm{i}}}>F_{Y}$. Then $v(Y)=v^{\text {up }}(Y) / 2+\lambda(Y) /(2 M)=1 / 2+$ $(M / 2) /(2 M)=3 / 4$. The line $1^{\text {up }}$ is incorporated in $Y$. Further, $v\left(Y_{i}\right)=v^{\text {up }}\left(Y_{i}\right) / 2+$ $\lambda\left(Y_{i}\right) /(2 M)<0 / 2+(M / 2) /(2 M)=1 / 4$. The line $1^{\text {up }}$ is not incorporated in $Y_{i}$, apart from its endpoints, which contribute measure 0 . If we take any $X$ with $v$-measure strictly between $1 / 4$ and $3 / 4$, then $Y_{i} \leqslant X$ for all $i$, but $Y>X$. Since $Y_{i}$ weakly converges to $Y$, weak convergence continuity is violated. Let us finally mention that this example does satisfy the simple continuity condition of Wakker (1990b).

\subsection{Example 4}

Suppose $v$ has a positive density function, denoted $\phi$. The integral of $\phi$ over any open neighborhood of $(0,1)$ is $\infty$. For any set that does not contain $(0,1)$ in its closure, $\phi$ is bounded, so that the integral of $\phi$ over that set is finite. Note that $v(Y)=0$ for $Y=[0, M]$ $\times\{1\}$, i.e., $Y \in L^{0}$ is the upper boundary of $[0, M] \times[0,1]$, corresponding with the degenerate probability distribution assigning probability 1 to the outcome 0 . All other $Y$ $\in L^{0}$ contain a point $(x, p)$ with $x>0$ and $p<1$, and thus contain an open neighborhood of $(0,1)$, and their $v$ value must be infinite. For this reason the preference relation $\geqslant$ cannot be derived from $v$ in the usual way. We derive $\geqslant$ from $v$ through comparison of difference sets, as follows. If $Y=[0, M] \times[0,1]$ and $Z \neq Y$, or if $Z=[0, M] \times\{1\}$ and $Y \neq Z$, then $Y>Z$. In all other cases, we define $Y>Z$ if and only if $v(Y \backslash Z)>v(Z \backslash Y)$; this is well defined because the closed difference sets $Y \backslash Z$ and $Z \backslash Y$ do not contain $(0,1)$, and their $v$ values are finite. The weak order $\geqslant$ on $L$ is well defined. We shall verify transitivity. Suppose $X \geqslant Y, Y \geqslant Z$. If any of these sets is $[0, M] \times[0,1]$ or $[0, M] \times\{1\}$, $X \geqslant Z$ is readily verified. In the other case, the preferences over $X, Y, Z$ are represented by the finite real numbers $v(X \backslash(X \cap Y \cap Z)), v(Y \backslash(X \cap Y \cap Z))$, and $v(Z(X \cap Y \cap Z)$ ), which implies $X \geqslant Z$. Transitivity has been established. Weak convergence continuity is satisfied, both if the limiting $Y$ is $[0, M] \times\{1\}$ (use countable additivity, and absolute continuity of $v$ with respect to $\lambda$ ), and if $Y \neq[0, M] \times\{1\}$. Also, stochastic dominance is satisfied. Although $\geqslant$ is not represented by a measure in the usual sense in this example, nevertheless irrelevance is satisfied; this follows from stochastic dominance if $[0, M] \times$ $\{1\}$ is the dispreferred lottery, and from substitution of $v$ otherwise. This example is related to example 25b in Wakker (1991a), where an additive representation is "driven to minus infinity" at minimal alternatives.

Similarly, an analogue of "driven to plus infinity" can occur. Then the integral of $\phi$ over every open neighborhood of $(M, 0)$ is infinite, the integral of $\phi$ over every set that does not contain $(M, 0)$ in its closure is finite, $[0, M] \times[0,1]$ has infinite $v$ value, and all other $Y \in L^{0}$ have finite $v$ value. So here $\geqslant$ can be defined by $Y \geqslant Z$ if and only if $v(Y)$ $\geq v(Z) ; v$, however, is not real-valued at $[0, M] \times[0,1]$. A condition to avoid the problems illustrated by this example is the second-order Archimedean axiom of Wakker (1991a; 1991b). This condition holds on the subspace of $1 / 3-1 / 3-1 / 3$ lotteries (a threedimensional rank-ordered space) if and only if the measure $v$ is bounded. 


\subsection{Example 5}

Let $v$ assign measure 1 to all sets that contain $(M / 2,1 / 2)$, and measure 0 to all sets that do not contain $(M / 2,1 / 2)$. Then $(M / 2,1 / 2)$ is an atom. Neither is $v$ absolutely continuous with respect to $\lambda$, nor is $\lambda$ with respect to $v$. The binary relation represented by $v$ does not satisfy weak continuity; neither does it satisfy stochastic dominance (it does satisfy weak stochastic dominance). It obviously satisfies the irrelevance axiom, and thus also the weaker "ordinal independence" axiom as used in Segal (1990, lemma 8).

\section{Conclusion}

This article has shown that the characterizations of the measure approach in Segal (1989, theorem 1) and Segal (1990, lemma 8) are not correct. First, the resulting measure may be infinite valued; second, the continuity conditions are not correct; and third and finally, countable additivity needs to be established. A correct theorem will be provided in Segal (1992). See also Chateauneuf (1990) and Chew and Wakker (1991, section 5).

\section{Notes}

1. Note that, for simple probability distributions $\left(p_{1}, x_{1} ; \ldots p_{n}, x_{n}\right)$, this provides a rewriting of the form given in the introduction. To wit, set $V(0, x)=V(p, 0)=0=u(0)$ for all $x, p$, and substitute $V\left(p_{1}+\ldots+p_{i}, x_{i}\right)$ $=v\left(\left[0, x_{i}\right] \times\left[1-\left(p_{1}+\ldots+p_{i}\right), 1\right]\right)$.

2. Actually, the weaker ordinal independence; however, this is equivalent to irrelevance for all purposes of this article.

3. Segal (1992), and Chew, Epstein, and Wakker (1991) prove that countable additivity must hold.

\section{References}

Chateauneuf, A. (1990). "On the Use of Comonotonicity in the Axiomatization of EURDP Theory for Arbitrary Consequences," CERMSEM, University of Paris I; extended abstract presented at Fifth International Conference on the Foundations and Applications of Utility, Risk and Decision Theory (FUR-90).

Chew, S.H. and L.G. Epstein. (1989). "A Unifying Approach to Axiomatic Non-Expected Utility Theories," Joumal of Economic Theory 49, 207-240.

Chew, S.H., L.G. Epstein, and P.P. Wakker. (1991). "A Unifying Approach to Axiomatic Non-Expected Utility Theories: Corrigenda,"Joumal of Economic Theory, forthcoming.

Chew, S.H. and P.P. Wakker. (1991). "Generalizing Choquet Expected Utility by Weakening Savage's SureThing Principle," University of California, Irvine Research Unit in Mathematical Behavioral Sciences, MBS 91-16, Irvine, CA, USA.

Fishburn, P.C. (1986). "The Axioms of Subjective Probability," Statistical Science 1, 335-358.

Gorman, W.M. (1968). "The Structure of Utility Functions," Review of Economic Studies 35, 367-390.

Green, J. and B. Jullien. (1988). "Ordinal Independence in Non-Linear Utility Theory," Journal of Risk and Uncertainty 1, 355-387. ("Erratum," 2 (1989, 119).)

Karni, E. and D. Schmeidler. (1990). "Utility Theory with Uncertainty." In W. Hildenbrand and H. Sonnenschein (eds.), Handbook of Mathematical Economics, Vol. 4. Amsterdam: North-Holland. 
Puppe, C. (1990). "The Irrelevance Axiom, Relative Utility and Choice under Risk," Department of Statistics and Mathematical Economics, University of Karlsruhe, Karlsruhe, Germany.

Quiggin, J. (1982). "A Theory of Anticipated Utility," Joumal of Economic Behaviour and Organization 3, $323-343$.

Quiggin, J. and P.P. Wakker. (1992). "The Axiomatic Basis of Anticipated Utility; A Clarification," University of Nijmegen, NICI, Nijmegen, The Netherlands.

Royden, H.L. (1963). Real Analysis. New York: MacMillan.

Schmeidler, D. (1989). "Subjective Probability and Expected Utility without Additivity," Econometrica 57, $571-587$.

Segal, U. (1986). "On Lexicographic Probability Relations," Mathematical Social Sciences 11, 195-199.

Segal, U. (1989). "Anticipated Utility: A Measure Representation Approach," Annals of Operations Research $19,359-373$.

Segal, U. (1990). "Two-Stage Lotteries without the Reduction Axiom," Econometrica 58, 349-377.

Segal, U. (1992). "The Measure Representation: A Correction," Joumal of Risk and Uncertainty, this issue.

Wakker, P.P. (1990a). "Continuity, Absolute Continuity, and Weak Convergence in Anticipated-Utility Representations," Fuqua School of Business, Duke University.

Wakker, P.P. (1990b). "Separating Marginal Utility and Probabilistic Risk Aversion," working paper FSB-9005, Fuqua School of Business, Duke University, Durham, NC, USA.

Wakker, P.P. (1991a). "Additive Representations on Rank-Ordered Sets. I. The Algebraic Approach," Joumal of Mathematical Psychology 35, 501-531.

Wakker, P.P. (1991b). "Additive Representations on Rank-Ordered Sets. II. The Topological Approach," Joumal of Mathematical Economics, forthcoming. 